\title{
KAT7 is a genetic vulnerability of acute myeloid leukemias driven by MLL rearrangements
}

\section{$\operatorname{AUTHOR}(S)$ :}

Au, Yan Zi; Gu, Muxin; De Braekeleer, Etienne; Gozdecka, Malgorzata; Aspris, Demetrios; Tarumoto, Yusuke; Cooper, Jonathan; ... Huntly, Brian J. P.; Vassiliou, George; Yusa, Kosuke

\section{CITATION:}

Au, Yan Zi ... [et al]. KAT7 is a genetic vulnerability of acute myeloid leukemias driven by MLL rearrangements. Leukemia 2020, 35(4): 10121022

\section{ISSUE DATE:}

2020-04

URL:

http://hdl.handle.net/2433/261905

\section{RIGHT:}

(C) The Author(s) 2020. This article is published with open access.; This article is licensed under a Creative Commons Attribution 4.0 International License, which permits use, sharing, adaptation, distribution and reproduction in any medium or format, as long as you give appropriate credit to the original author(s) and the source, provide a link to the Creative Commons license, and indicate if changes were made. The images or other third party material in this article are included in the article's Creative Commons license, unless indicated otherwise in a credit line to the material. If material is not included in the article's Creative Commons license and your intended use is not permitted by statutory regulation or exceeds the permitted use, you will need to obtain permission directly from the copyright holder. To view a copy of this license, visit http://creativecommons.org/license.. 


\title{
ARTICLE
}

\section{Acute myeloid leukemia}

\section{KAT7 is a genetic vulnerability of acute myeloid leukemias driven by $M L L$ rearrangements}

\author{
Yan Zi Au ${ }^{1,2,9} \cdot$ Muxin $\mathrm{Gu}^{2}$ - Etienne De Braekeleer ${ }^{2}$ - Malgorzata Gozdecka ${ }^{2,3}$ - Demetrios Aspris ${ }^{2}$. \\ Yusuke Tarumoto $\mathbb{D}^{4} \cdot$ Jonathan $\mathrm{Cooper}^{2} \cdot$ Jason Yu $\mathbb{D}^{1,10}$. Swee Hoe Ong $\mathbb{D}^{1} \cdot \mathrm{Xi} \mathrm{Chen}^{5}$. \\ Konstantinos Tzelepis $\mathbb{( D}^{2,6} \cdot$ Brian J. P. Huntly $\mathbb{1}^{3,7,8} \cdot$ George Vassiliou $\mathbb{C}^{2,3,7} \cdot$ Kosuke Yusa $\mathbb{D}^{1,4}$
}

Received: 6 August 2019 / Revised: 15 July 2020 / Accepted: 22 July 2020

(c) The Author(s) 2020. This article is published with open access

\begin{abstract}
Histone acetyltransferases (HATs) catalyze the transfer of an acetyl group from acetyl-CoA to lysine residues of histones and play a central role in transcriptional regulation in diverse biological processes. Dysregulation of HAT activity can lead to human diseases including developmental disorders and cancer. Through genome-wide CRISPR-Cas9 screens, we identified several HATs of the MYST family as fitness genes for acute myeloid leukemia (AML). Here we investigate the essentiality of lysine acetyltransferase KAT7 in AMLs driven by the MLL-X gene fusions. We found that KAT7 loss leads to a rapid and complete loss of both H3K14ac and H4K12ac marks, in association with reduced proliferation, increased apoptosis, and differentiation of AML cells. Acetyltransferase activity of KAT7 is essential for the proliferation of these cells. Mechanistically, our data propose that acetylated histones provide a platform for the recruitment of MLL-fusion-associated adaptor proteins such as BRD4 and AF4 to gene promoters. Upon KAT7 loss, these factors together with RNA polymerase II rapidly dissociate from several MLL-fusion target genes that are essential for AML cell proliferation, including MEISI, $P B X 3$, and SENP6. Our findings reveal that KAT7 is a plausible therapeutic target for this poor prognosis AML subtype.
\end{abstract}

These authors contributed equally: Yan $\mathrm{Zi} \mathrm{Au,} \mathrm{Muxin} \mathrm{Gu}$

Supplementary information The online version of this article (https:// doi.org/10.1038/s41375-020-1001-z) contains supplementary material, which is available to authorized users.

George Vassiliou gsv20@sanger.ac.uk

$\triangle$ Kosuke Yusa k.yusa@infront.kyoto-u.ac.jp

1 Stem Cell Genetics, Wellcome Sanger Institute, Hinxton, Cambridge, UK

2 Haematological Cancer Genetics, Wellcome Sanger Institute, Hinxton, Cambridge, UK

3 Wellcome Trust-MRC Stem Cell Institute, Cambridge Biomedical Campus, University of Cambridge, Cambridge, UK

4 Stem Cell Genetics, Institute for Frontier Life and Medical Sciences, Kyoto University, Kyoto, Japan

\section{Introduction}

Acute myeloid leukemia (AML) is an aggressive malignancy of haematopoietic stem and progenitor cells. Translocations involving the mixed lineage leukemia gene (MLL or KMT2A) characterize an aggressive subtype of the disease and are associated with an intermediate or poor prognosis [1-3]. Currently, chemotherapy based on a combination of anthracyclines and purine analogs is the standard of care for AML,

5 Gene Expression Genomics, Wellcome Sanger Institute, Hinxton, Cambridge, UK

6 Gurdon Institute, University of Cambridge, Tennis Court Road, Cambridge, UK

7 Department of Haematology, Cambridge University Hospitals NHS Trust, Cambridge, UK

8 Cambridge Institute for Medical Research, University of Cambridge, Cambridge, UK

9 Present address: Dana-Farber Cancer Institute, Boston, MA, USA

10 Present address: Department of Cell Biology, The Francis Crick Institute, London, UK 
yet patients with leukemias driven by $M L L-X$ gene fusions commonly become refractory to such treatments [4]. Advances in our understanding of the molecular pathogenesis of acute leukemias driven by $M L L-X$ fusion oncogenes have led to the development of new drugs, including inhibitors of bromodomain proteins [5-7], DOT1L [8, 9], and MENIN [10]; however, none of these have demonstrated a significant clinical benefit for patients as yet. In order to identify new therapeutic approaches in AML, we and others have performed genome-wide CRISPR-Cas9 fitness screens in different subtypes of the disease including the $M L L-X$ subtype [11, 12]. Through our own screen data, we recently showed that GCN5 (KAT2A) and SRPK1 are novel vulnerability in AML and that sensitivity to SRPK1 inhibition is particularly associated with AML driven by $M L L-X$ fusion oncogenes [11, 13]. Systematic detailed analysis of our CRISPR screen dataset revealed that several members of the MYST family of histone acetyltransferases (HATs) are required for the survival of AML cell lines, with KAT7 displaying strong essentiality for AMLs driven by $M L L-X$ oncogenes.

HATs are classified based on structural and sequence homology into distinct groups including the p300/CBP, MYST, and GCN5 families [14]. They play a central role in transcriptional regulation through their function to catalyze the transfer of acetyl groups from acetyl-CoA to the $\varepsilon$-amino group of lysine residues of histones. Dysregulation of HATs is known to be associated with human diseases including developmental disorders and cancer [14-16]. The MYST family of HATs, characterized by their conserved MYST catalytic domain, includes KAT5 (TIP60), KAT6A (MOZ and MYST3), KAT6B (MORF and MYST4), KAT7 (HBO1 and MYST2), and KAT8 (MOF) $[15,16]$. While $K A T 6 A$ and $K A T 6 B$ are known targets of chromosomal translocations that drive AML [17-21] and KAT8 can play a role in some cases of AML [22], it is currently not known whether KAT7 plays a role in leukemogenesis. As KAT7 lacks chromatin binding domains, it relies on forming complexes with a scaffold protein (JADE or BRPF), along with EAF6 and ING4/5, for efficient histone acetylation [23]. The choice of the scaffold protein primarily determines target histones, namely $\mathrm{H} 3$ or H4. The KAT7-BRPF acetylates histone H3 tails (K14 and K23) [23-26], whereas KAT7-JADE complexes acetylates histone $\mathrm{H} 4$ tails (K5, K8, and K12) [27], respectively. In development, abolishing KAT7 results in major reduction of $\mathrm{H} 3 \mathrm{~K} 14 \mathrm{ac}$ in both mouse fetal liver erythroblasts [24] and mouse embryo [25]; the latter associates with lethality.

Here we investigate the molecular mechanism underlying the requirement of KAT7 in $M L L-X$-driven AMLs. We report that loss of KAT7 leads to reduced proliferation and enhanced apoptosis and differentiation. We also show that KAT7 is essential for maintaining the transcriptional program driven by MLL-AF9, through the recruitment of BRD4 and other MLL-fusion associated proteins such as AFF1 to the promoters of a subset of MLL-AF9 target genes. Together, our findings propose KAT7 as a plausible therapeutic target in $M L L-X$ AML and also provide novel mechanistic insights into the molecular basis of MLL-Xdriven transcriptional dysregulation.

\section{Materials and methods}

\section{Cell culture}

Human cell lines (MOLM-13, MV4-11, THP-1, HL-6, K562, Nomo-1, OCI-AML2, OCI-AML3, MA9-ITD, and MA9RAS) and a mouse cell line (RN2) were used in this study. All these cell lines stably express Cas9 (refs. [11, 28, 29]) and are confirmed to be mycoplasma negative. Details in Supplementary Methods.

\section{Proliferation assay}

Cells were transduced with gRNA-expressing lentivirus and the percentage of BFP-positive (knock-out) cells was determined every 2 days between day 4 and day 14 using flow cytometry. gRNA sequences used were listed in Table S1. Details in Supplementary Methods.

\section{Differentiation and apoptosis assays}

Cells were transduced with gRNA-expressing lentivirus and BFP-positive (knock-out) cells were collected by cell sorting. For differentiation analysis, cells were stained with APC-conjugated CD11b (eBioscience) 7 days post transduction. For apoptosis assays, cells were analyzed 9 days post transduction using Annexin V Apoptosis Detection Kit APC (eBioscience). Details in Supplementary Methods.

\section{Generation of auxin inducible degron (AID) KAT7 protein degradation system and treatment with indole-3-acetic acid (IAA)}

MOLM-13 cells were used to generate cells expressing KAT7-AID. The resulting cells were treated with $500 \mu \mathrm{M}$ IAA for the duration specified for each assay. Details in Supplementary Methods.

\section{Western blot analysis}

Western blot analysis was performed according to the manufacturer's instructions. Antibodies used were listed in Table S2. Details in Supplementary Methods. 
A

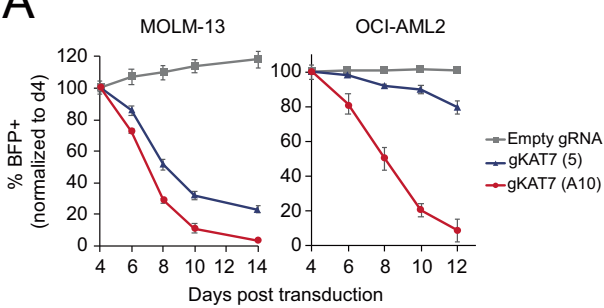

C

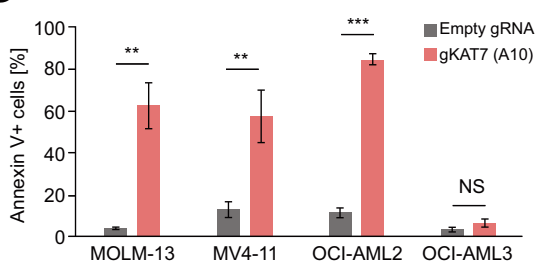

Fig. 1 Loss of KAT7 exhibits antileukemic effects in vitro and in vivo. a Proliferation of $K A T 7 \mathrm{KO}$ using two sgRNAs or empty control in $M L L-X$ AML cell lines. Percentages of BFP-positive (KAT7$\mathrm{KO})$ cells were assayed at the indicated time point and were normalized to day 4. Data are shown as mean $\pm \operatorname{SD}(n=3)$. b CD11b staining in WT (empty gRNA) and KAT7 KO (gKAT7-A10) cells 7 days post

\section{In vivo mouse work}

MOLM-13-Cas9-luc cells [11] were transduced with gRNA-expressing lentivirus and BFP-positive cells were sorted 2 days post transduction. On the following day, $5 \times$ $10^{5}$ cells were injected into NSGW41 male mice. All animal studies were carried out at the Wellcome Sanger Institute under UK Home Office License PBF095404. Details in Supplementary Methods.

\section{ChIP-seq and ChIP-qPCR}

MOLM-13, OCI-AML3, THP-1, and OCI-AML2 were used for ChIP-seq and/or ChIP-qPCR analysis using antibodies listed in Table S2 and qPCR primers listed in Table S3. Details in Supplementary Methods.

\section{RNA extraction and RNA-seq processing}

RNA was extracted from AML cells with RNeasy Plus Mini Kit (Qiagen) according to manufacturer's instructions and sequenced on Illumina HiSeq v4 platform with 75-bp paired-end sequencing. Data processing and analysis were detailed in Supplementary Methods.

\section{Statistical analysis}

Normal distribution was first tested using $F$-test. Student's $t$-test was used for statistical testing unless stated otherwise.
B

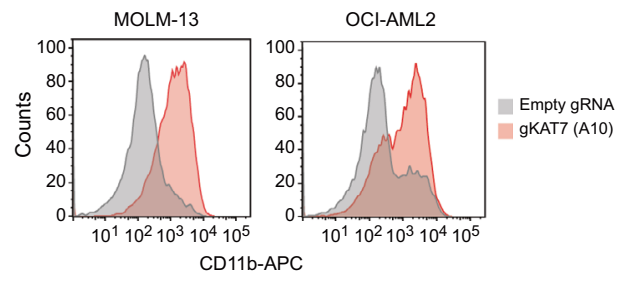

D

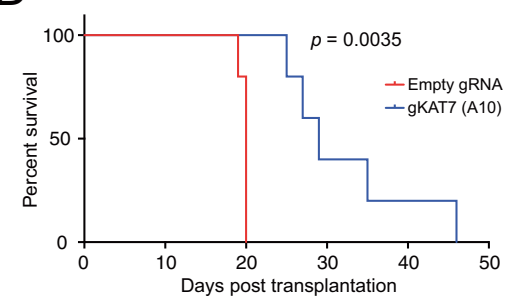

transduction. c Annexin V/PI staining of WT (Empty) and KAT7 KO (gKAT7-A10) cells 9 days post transduction. Data are shown as mean $\pm \mathrm{SD}(n=3)$. Two-tailed $t$-test was performed (N.S., not significant; $* * P<0.01 ; * * * P<0.001)$. d Xenograft analysis of $K A T 7-\mathrm{KO}$ MOLM-13. Kaplan-Meier survival analysis were performed $(n=5$ in each arm).

Mean was calculated from at least three replicates, as indicated in each figure, and error bars represent the standard deviation. $P$ values $\leq 0.05$ were considered statistically significant.

\section{Results}

\section{Loss of KAT7 induces myeloid differentiation and apoptosis in AML cell lines with MLL fusion oncoprotein}

We previously cataloged fitness genes in five AML cell lines (MOLM-13, MV4-11, OCI-AML2, OCI-AML3, and HL-60) by genome-scale CRISPR-KO screening and characterized promising therapeutic targets including the histone lysine acetyltransferase KAT2A and SRPK1 [11, 13]. We further looked into genes encoding histone modifying enzymes and found that several histone acetyl transferases (HATs) were required for AML cell proliferation (Supplementary Fig. S1A). Notably, two of the MYST family HATs, KAT6A, and KAT7, were essential for AML cell lines driven by $M L L-X$ fusion genes: MOLM-13 (driven by MLL-AF9), MV4-11 (MLL-AF4), and OCI-AML2 (MLLAF6). In order to validate these fitness defects, we first investigated the effects on proliferation in MOLM-13 and MV4-11 by gRNA-mediated KO and found that KAT7 loss exhibited a consistent strong anti-proliferative effect in both cell lines (Supplementary Fig. S1B). We then tested the 
Fig. 2 Catalytic activity of KAT7 is required for leukemic maintenance. a Western blot analysis of potential KAT7 acetylation sites on $\mathrm{H} 3$ and $\mathrm{H} 4$ in MOLM-13 cells. b Western blot analysis of the KAT7 acetylation sites in MOLM-13 expressing exogenous WTKAT7 or HAT-dead KAT7 (E508Q) with or without endogenous KAT7 KO by lentiviral gRNA expression. Proliferation (c) and CD11b staining (d) of MOLM-13 expressing exogenous KAT7 WT or E508Q mutant with endogenous KAT7 disrupted by gKAT7. Data are shown as mean $\pm \mathrm{SD}(n=3)$.
A
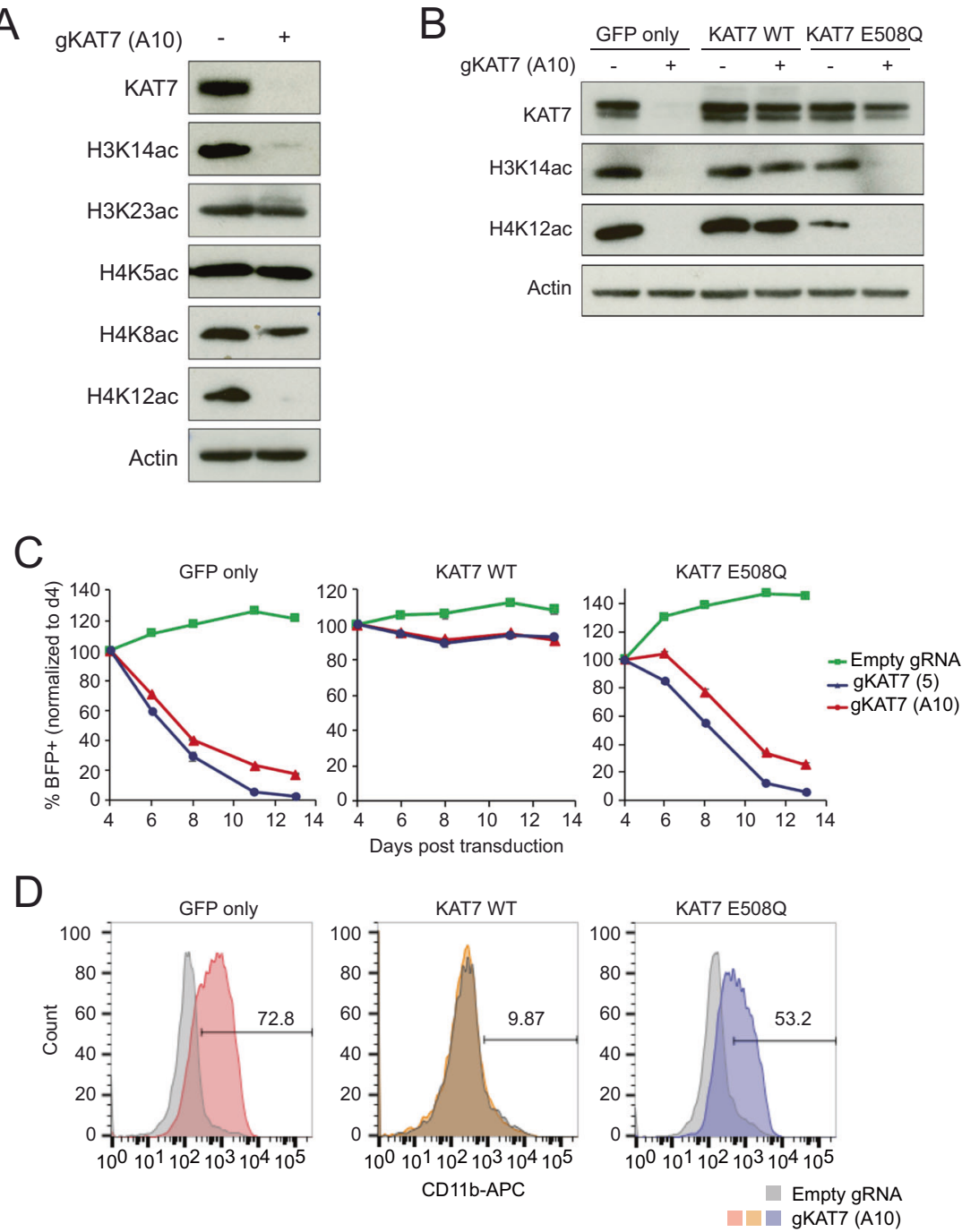

impact of genetic disruption of $K A T 7$ on proliferation of all cell lines used in our CRISPR-KO screens and 3 additional human leukemia cell lines, and found that, consistent with MOLM-13 and MV4-11, KAT7 loss decreased the proliferation of other $M L L-X$ cell lines, namely OCI-AML2, THP-1 (MLL-AF9), and Nomo-1 (MLL-AF9), but not of MLL-WT cell lines such as OCI-AML3, HL-60, and K562 (Fig. 1a, Supplementary Fig. S1C, D). KAT7 dependency of $M L L-X$ leukemic cells was also confirmed in an independently generated THP-1 Cas9 line [28], human CD34+ cells transformed by MLL-AF9 (refs. [28, 30]) and a mouse AML cell line bearing MLL-AF9 (refs. [6, 29]) (Supplementary Fig. S1E-G). The proliferation phenotype was associated with increased expression of the myeloid differentiation marker CD11b in MOLM-13 and OCI-AML2 (Fig. 1b, Supplementary Fig. S1H). KAT7 loss also led to an increased apoptosis in $M L L-X$ cell lines (Fig. 1c). Collectively, these results indicate that KAT7 is required for in vitro survival and proliferation of leukemic cells driven by $M L L-X$ fusion oncogenes. To assess if KAT7 inactivation has also anti-proliferative effects in vivo, we injected MOLM-13 cells previously transduced with a KAT7 gRNA (KAT7-KO) into NSGW41 male mice. Mice transplanted with KAT7-KO MOLM-13 cells showed significantly slower AML progression and increased survival, compared to those injected with MOLM-13 expressing a control gRNA (Fig. 1d, Supplementary Fig. S1I), indicating the requirement of KAT7 for in vivo proliferation.

\section{KAT7 regulates global H3K14 and H4K12 acetylation}

KAT7 is known to form two distinct HAT complexes that acetylate either $\mathrm{H} 3$ or $\mathrm{H} 4$ (ref. [23]). We therefore investigated the acetylation status of $\mathrm{H} 3$ and $\mathrm{H} 4$ tails in KAT7 KO MOLM-13 cells. Among the potential KAT7 acetylation sites, acetylation of Lysine-14 residue of $\mathrm{H} 3$ (K3K14ac) and Lysine-12 of $\mathrm{H} 4$ (H4K12ac) were completely abolished upon KAT7 KO, whereas other acetylation sites were not 
Fig. 3 Transcriptomic profiling of $\mathrm{KAT} 7 \mathrm{KO}$ in MOLM-13 (MLL-AF9), OCIAML3 (MLL WT). a Numbers of differentially expressed (DE) genes in MOLM-13 and OCIAML 3 on day 3 and day 5 after gRNA-mediated $K A T 7 \mathrm{KO}$. b GSEA on day 3 (top) and day 5 (bottom) transcriptomes in MOLM-13. Gene sets consisting of genes upregulated upon myeloid differentiation (left), MLL-AF9 target genes (center), or MLL-AF9 spreading genes (right; Supplementary Table S4) were used. c MA plot of day 3 MOLM-13 comparing KAT7 WT and KO. Black, DE genes (adj. $P<0.05$ ); yellow, MLLAF9 spreading genes. Key MLL-AF9 spreading genes, namely $M E I S 1, P B X 3, J M J D 1 C$, SENP6, and $M E F 2 C$ were highlighted.
A

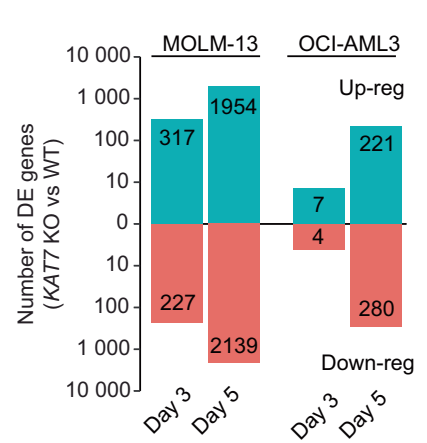

C

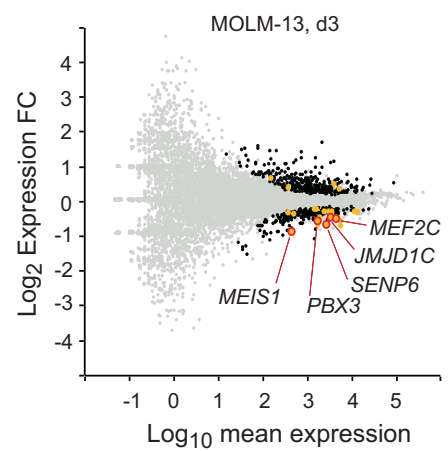

B

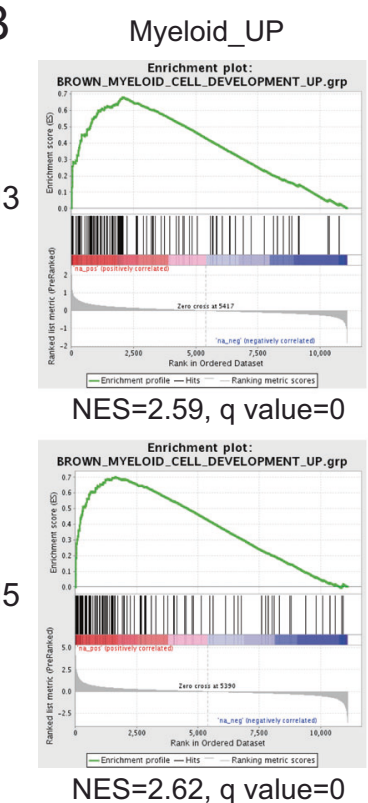

MLL-AF9_Spreading Enrichment plot: 114_Spreading_KAT 7bound.grp

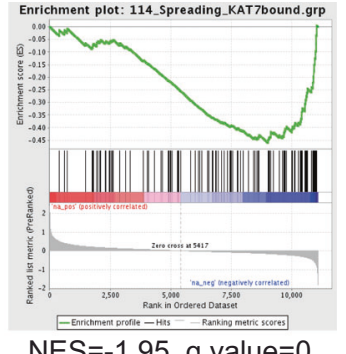

NES $=-1.95$, q value $=0$

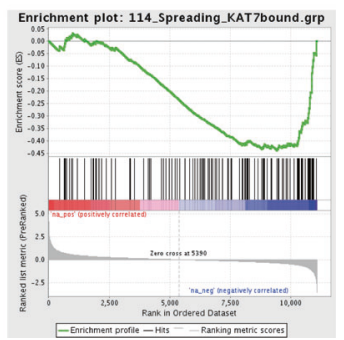

$\mathrm{NES}=2.62, \mathrm{q}$ value $=0$ affected (Fig. 2a). We additionally investigated major histone marks for active transcription, namely $\mathrm{H} 3 \mathrm{~K} 4 \mathrm{me} 3$, $\mathrm{H} 3 \mathrm{~K} 9 \mathrm{ac}$, and H3K27ac, and found that KAT7 loss did not affect the global level of these marks (Fig. S2A). Interestingly, global loss of H3K14ac and H4K12ac upon KAT7 loss was observed in all AML cell lines tested, irrespective of the $M L L$ translocation status or proliferation phenotype (Fig. S2B).

\section{Acetyltransferase activity of KAT7 is essential for leukemic maintenance}

Since both $M L L-X$ and $M L L$-WT AML cell lines showed globally reduced $\mathrm{H} 3 \mathrm{~K} 14$ and $\mathrm{H} 4 \mathrm{~K} 12$ acetylation upon $K A T 7 K O$ but only $M L L-X$ AML cell lines showed reduced proliferation, we considered that noncatalytic roles of KAT7 complexes may have played a role in the observed proliferative defect. To rule out such possibility, we performed cDNA reconstitution experiments using gRNA-resistant WT and HAT-dead KAT7 cDNAs. For the HAT-dead KAT7 mutant, we utilized a previously characterized
E508Q MYST domain mutant [31]. To replace endogenous KAT7 with a cDNA-derived protein, we first expressed a gRNA-resistant KAT7 cDNA and then knocked out the endogenous KAT7 by lentiviral transduction of KAT7 gRNA. Western blot analyses showed that control (GFP only) MOLM-13 cells lost KAT7 as well as acetylation marks on both H3K14 and H4K12 upon the endogenous KAT7 KO, whereas cells carrying the WT KAT7 cDNA retained KAT7 protein and both acetylation marks, even after gRNA-targeting of the endogenous KAT7 gene (Fig. 2b), indicating the appropriate reconstitution of KAT7 activity. In contrast, cells expressing HAT-dead E508Q mutant KAT7 protein completely lost both acetylation marks after endogenous KAT7 KO (Fig. 2b). We noted that cells expressing both endogenous KAT7 and exogenous HAT-dead KAT7 showed considerably reduced H4K12ac, although $\mathrm{H} 3 \mathrm{~K} 14 \mathrm{ac}$ was maintained at the level similar to that of the control cells. This suggest a dominant negative effect of HAT-dead KAT7 protein; however, proliferation of MOLM-13 cells expressing either exogenous KAT7 was not significantly different (Fig. S2C). We then analyzed 
Fig. 4 Transcriptomic profiling of the acute phase of KAT7 depletion in MOLM-13 using the degron system. a Time-course western blot analysis of KAT7 and H3K14ac in IAA-treated MOLM-13 KAT7-AID cells. b Cell proliferation of IAA-treated KAT7-AID and parental MOLM-13. Data are shown as mean \pm S.D. $(n=3)$. Two-tailed $t$-test was performed $(* * * P \leq$ 0.001). c GSEA on transcriptome of MOLM-13 KAT7-AID cells treated with IAA for 2- (top) or $4 \mathrm{~h}$ (bottom). The same genesets as Fig. 3b were used.
A

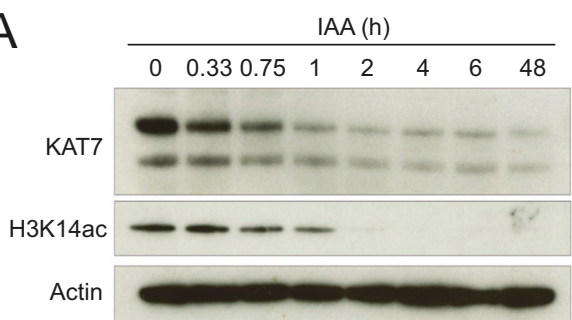

B

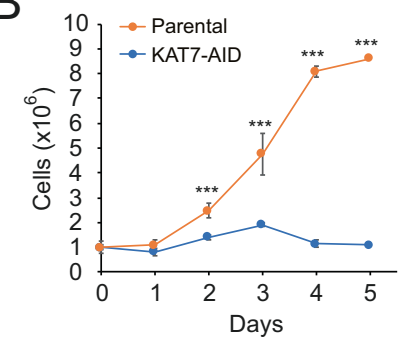

C

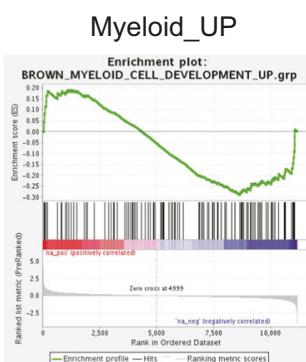

Not significant

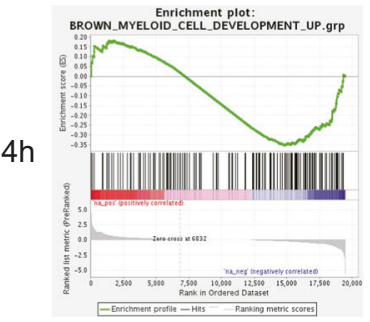

Not significant

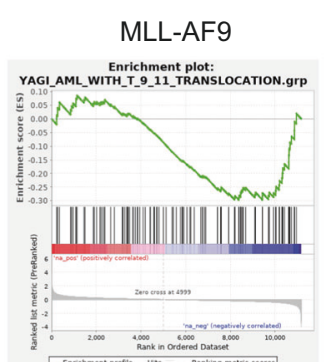

Not significant

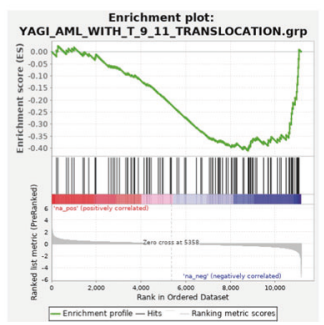

NES $=-1.53$, q value $=0.005$

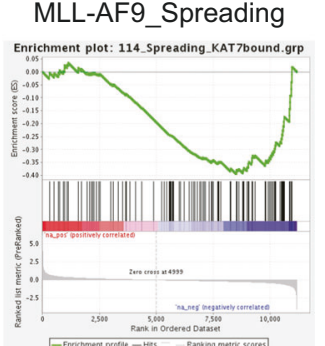

NES $=-1.50$, q value $=0.015$

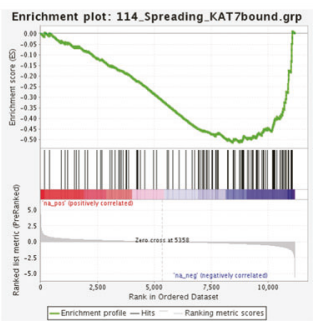

$\mathrm{NES}=-1.97, \mathrm{q}$ value $=0$ proliferation of KAT7-reconstituted cells and found that KAT7-WT-expressing cells did not show a proliferation defect, ruling out the possibility that off-target effects of $K A T 7 \mathrm{gRNAs}$ might have been responsible for the observed phenotype (Fig. 2c, center). In sharp contrast, the cells expressing HAT-dead KAT7 showed proliferation defect (Fig. 2c, right), similar to that in endogenous KAT7 $\mathrm{KO}$ cells (Fig. 2c, left). This proliferation defect was associated with increased CD11b expression (Fig. 2d). Thus, the cells expressing HAT-dead KAT7 phenocopied the endogenous KAT7 KO cells in histone acetylation, proliferation, and differentiation. These results strongly indicate that KAT7mediated acetylation plays crucial roles in the maintenance of the leukemic program driven by the MLL-X fusion protein.

\section{KAT7 loss leads to downregulation of a specific set of MLL-AF9 target genes}

In order to analyze transcriptomic changes upon $K A T 7 \mathrm{KO}$, we performed RNA-seq analyses of cells transduced with lentivirus expressing KAT7 or control gRNA (Supplementary Dataset 1). We chose MOLM-13 and OCI-AML3 as the $M L L-X$ positive and negative cell lines, respectively, in this analysis. On day 3 after transduction, we observed a total of 544 differentially expressed (DE) genes (317 upand 227 down-regulation) in MOLM-13 (Fig. 3a). The number of DE genes increased further on day 5 to 4093 (1954 up- and 2139 down-regulation). In contrast, the KAT7-independent OCI-AML3 showed only 11 genes on day 3 and 501 on day 5. Consistent with myeloid differentiation observed by flow cytometry (CD11b upregulation, Fig. 1b), gene set enrichment analysis (GSEA) revealed that a gene set consisting of genes upregulated upon myeloid differentiation was significantly enriched on days 3 and 5 (Fig. 3b, left). Concomitantly, the MLL-AF9 target gene set was significantly depleted on days 3 and 5 (Fig. 3b, center).

Through inspection of differentially expressed genes in MOLM-13 on day 3, we identified that a small fraction of the MLL-AF9 target genes, most notably MEIS1, PBX3, $J M J D 1 C$, SENP6, and $M E F 2 C$, were significantly downregulated (Fig. 3c). Together with HOXA9, MEIS1, and PBX3 are known to form a complex and promote transcription of HOXA target genes [32]. JMJD1C and MEF2C are also known to play an important role in MLL-AF9induced leukemogenesis [28, 33]. In addition, these genes have recently been shown to be among genes, of which 
Fig. 5 KAT7 binds to and is required for expression of a subset of MLL-AF9 targets. Average occupancy of KAT7 per gene per base at the TSS of highly active genes $(>10$ FPKM), active genes $(>0, \leq 10$ FPKM) and inactive genes $(\mathrm{FPKM}=0)$ in MOLM-13 (a) and OCI-AML3 (b). Comparison between KAT7 promoter occupancy and gene expression changes 3 days after KAT7 KO in MOLM-13 (c) and OCI-AML3 (d) for MLL-AF9 spreading genes. DE genes are highlighted in blue (up) or red (down).
A
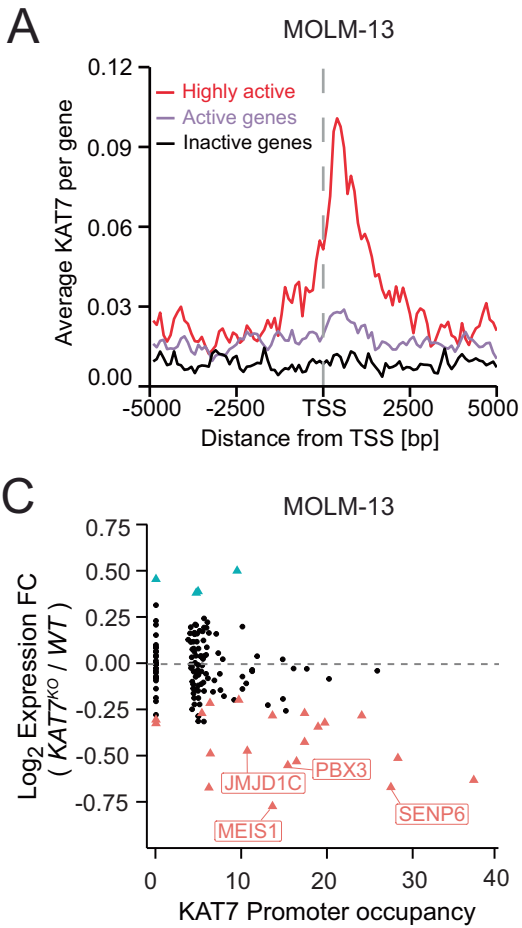

B
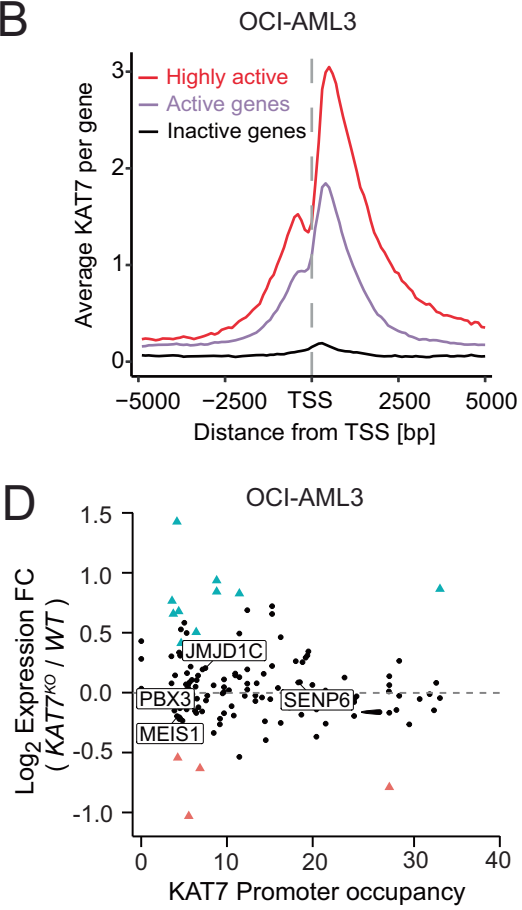

MLL-AF9 binding is not limited to their promoter region but spreads into their gene body [34]. These MLL-X oncoprotein "spreading" genes are collectively more susceptible to DOT1L inhibition than MLL-X "non-spreading" genes [34], indicating that the spreading genes comprise the core downstream network of the $M L L-X$-driven leukemic program. We further performed GSEA on the expression profiles with a gene set consisting of protein-coding KAT7bound MLL-AF9 spreading genes (see below, Supplementary Table S4) and found significant enrichment (Fig. 3b, right). These enrichment was not observed in the $M L L-\mathrm{WT}$, KAT7-independent OCI-AML3 cell line.

In order to more accurately capture transcriptomic changes immediately after KAT7 loss, we employed the auxin-inducible degron (AID) system [35]. For this, we first stably expressed KAT7-AID and then knocked out the endogenous KAT7 with gRNA. We confirmed that proliferation of MOLM-13 KAT7-AID was similar to that of parental MOLM-13 cells (Supplementary Fig. S3A), indicating that AID-tagged KAT7 is functional. We then introduced OsTIR1 and treated cells with auxin IAA to deplete KAT7 protein. The KAT7 protein level rapidly decreased and plateaued $2 \mathrm{~h}$ after treatment (Fig. 4a). This was associated with complete loss of H3K14ac over the same time course (Fig. 4a). Cells treated with IAA showed decreased proliferation, increased levels of apoptosis and myeloid differentiation (CD11b) at $48 \mathrm{~h}$ (Fig. 4b, Supplementary Fig. S3B, C). Therefore, the phenotype after IAA treatment faithfully mirrored those seen with CRISPRmediated KAT7 KO (Figs. 1a-c, 2a), indicating that the
KAT7-AID system can be used to study the direct effects of KAT7 loss.

We performed RNA-seq analysis using this MOLM-13 KAT7-AID line (Supplementary Dataset 1) and subsequently GSEA. After both 2- and 4-h of treatment with IAA, there was no significant myeloid gene upregulation (Fig. 4c, left). In contrast, enrichment of MLL-AF9 spreading genes was detectable as early as $2 \mathrm{~h}$, which preceded enrichment of the entire MLL-AF9 target gene set (Fig. 4c, center and right). These results indicate that KAT7 loss disrupts a leukemia maintenance program through downregulation of the MLL-AF9 spreading genes and subsequently leads to myeloid differentiation.

\section{KAT7 binds to promoters of active genes, especially a subset of MLL-AF9 spreading genes}

We next investigated the genomic location of KAT7 binding sites using chromatin-immunoprecipitation followed by sequencing (ChIP-seq) analysis (Supplementary Dataset 2). We again chose MOLM-13 and OCI-AML3 for this analysis and identified 9214 and 60153 KAT7 peaks, respectively. KAT7 was most enriched at promoters in both cell lines; $63.3 \%$ of the KAT7 signals in MOLM-13 and $48.5 \%$ in OCI-AML3 were found in promoter regions $( \pm 2 \mathrm{~kb}$ from TSSs) (Supplementary Fig. 5a, b, S4A). Consistent with previously reported ChIP-seq studies [16, 23, 24], KAT7 occupancy in the AML cell lines is significantly correlated with the expression levels of target genes (Fig. 5a, b, Supplementary Fig. S4B). We then focused our analysis on 
the MLL-AF9 spreading gene set. These genes are expressed not only in the MLL-AF9-bearing MOLM-13 but also in $M L L$-WT OCI-AML3 with a significant correlation of their expression levels between the two cell lines (Fig. S4C, $r^{2}=0.80$ ). As KAT7 marks expressed genes, the majority of the MLL-AF9 spreading genes were bound by KAT7 in both cell lines (Fig. S4D). We then compared expression levels and KAT7 promoter occupancy of the spreading genes (Supplementary Fig. S4E) and found that, consistent with the genome-wide patterns, OCI-AML3 showed good correlation between the two parameters (Supplementary Fig. S4E, right); however, MOLM-13 did not show clear correlation, but KAT7 rather seemed to be highly bound to a small fraction of the MLL-AF9 spreading genes (Supplementary Fig. S4E, left). We further compared KAT7 promoter occupancy with expression fold changes on day 3 after KAT7 KO and found that in MOLM-13 genes with higher KAT7 occupancy were more susceptible to and downregulated upon KAT7 loss (Fig. 5c). In particular, MEIS1, PBX3, JMJD1C, and SENP6, which were significantly downregulated on day 3 (Fig. 3c), showed particularly high KAT7 promoter occupancy (Fig. 5c). There were significant differences in KAT7 promoter occupancy between the downregulated $(n=20)$ and the upregulated $(n=4)$ or not-differentially expressed $(n=126)$ genes (Supplementary Fig. S4F), and KAT7 was markedly enriched in promoters of the KAT7-responsive MLLAF9 spreading genes (Supplementary Fig. S4G). In sharp contrast and consistent with its KAT7 independence, in OCI-AML3 KAT7 loss did not show a significant impact on expression on day 3, even for genes with KAT7 promoter occupancy comparable to those downregulated in MOLM13 (Fig. 5d, Supplementary Fig. S4F). In both cell lines, for non-MLL-AF9 target genes, there was no significant difference in KAT7 promoter occupancy between upregulated, downregulated and not-differentially expressed genes (Supplementary Fig. S5A-D). Taken together, these results suggest that although KAT7 is not required for general transcription in AML, KAT7, and its acetylase activity are required for MLL-AF9 oncoprotein to sufficiently upregulate and maintain expression of a specific set of the MLLAF9 spreading genes.

\section{MLL-fusion associated machineries are evicted from their target loci upon KAT7 loss}

To study the consequence of KAT7 loss at the chromatin level, we utilized the KAT7-degron system (Fig. 4, Supplementary Fig. S3) and performed ChIP followed by quantitative PCR (ChIP-qPCR) analysis. We first investigated whether KAT7 is required for MLL-AF9 fusion protein to maintain its binding to the promoters of key MLL-AF9 spreading genes, namely JMJD1C, SENP6, $P B X 3$, and MEIS1, which were downregulated significantly on day 3 after KAT7 KO (Fig. 3c) and bound by KAT7 with markedly high occupancy (Fig. 5c). ChIP-qPCR analysis using antibodies against MLL N-terminal and AF9 C-terminal regions $24 \mathrm{~h}$ after IAA treatment showed no significant changes in their occupancy at the promoter region (Supplementary Fig. S6A, B). This observation is consistent with previous findings that MLL-AF9 recruitment is dependent on interactions with MENIN [36], LEDGF [37], and the PAF complex [38].

We next investigated the binding of the histone acetylation "reader" BRD4 at gene promoters. BRD4 is a key interactor of MLL-fusion proteins and a target of antileukemic drugs $[5,6]$. Globally, BRD4 occupancy at promoter regions is highly correlated with KAT7 occupancy (Supplementary Fig. S6C). At the selected key MLL-AF9 spreading genes, KAT7, MLL-AF9, and BRD4 highly co-localized (Supplementary Fig. S6D). Upon KAT7 depletion, BRD4 markedly dissociated from the promoters of the key spreading genes (Fig. 6a). This was associated with dissociation of AFF1, a scaffold protein of the super elongation complex (SEC), from these promoters (Fig. 6b). In addition, RNA polymerase II Serine 5 phosphorylation occupancy was also dramatically reduced at these loci (Fig. 6c). BRD4 dissociation from the promoters was also confirmed in THP-1
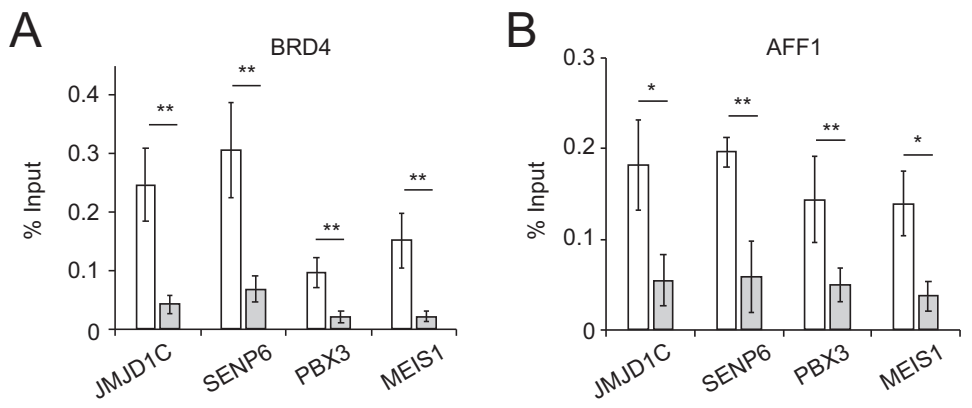

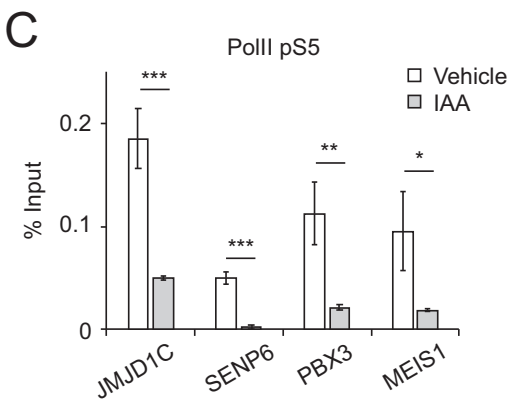

the promoter region of the key MLL-AF9 spreading genes. Data are shown as mean \pm S.D. $(n=3)$. Two-tailed $t$-test was performed ( ${ }^{*} P \leq$ $0.05 ; * * P \leq 0.01 ; * * * P \leq 0.001)$.
Fig. 6 KAT7-dependent recruitment of BRD4 and SEC complex to a subset of MLL-AF9 spreading genes. ChIP-qPCR analysis of BRD4 (a), AFF1 (b) and pS5 Pol II (c) after $24 \mathrm{~h}$ of IAA treatment at 
and OCI-AML2 (Supplementary Fig. S6E, F). These results suggest that KAT7-mediated histone acetylation serves as a scaffold for BRD4 binding to chromatin, which is required for sustained recruitment of transcriptional activators (Supplementary Fig. S7).

\section{Discussion}

Despite advances in understanding its genomics and molecular pathogenesis, AML remains lethal to the majority of sufferers [39] and anti-AML mainstream therapies have not changed significantly for several decades. Among different AML subtypes, cases driven by $M L L-X$ fusion genes continue to represent an intermediate and poor prognostic category [1-3] and despite recent developments in the field $[5,6,8,10,40]$ clinical progress is still lacking, emphasizing the need for new therapies. Here, we demonstrate that KAT7 represents a promising novel therapeutic target for $M L L-X$ AML and provide insights into its function in the maintenance of these leukemias.

KAT7-containing complexes have been shown to acetylates lysine residues of histone $\mathrm{H} 3$ and $\mathrm{H} 4$ tails. Specificity for the target histone is determined by the scaffold subunits, BRPF and JADE. In in vitro HAT assays using nucleosomes, BRPF-containing complex acetylates $\mathrm{H} 3 \mathrm{~K} 14$ and K23, while JADE-containing complex acetylates H4K5, K8, and K12 (ref. [23]). These scaffold subunits can also form a HAT complex with KAT6A and KAT6B and show a similar specificity against $\mathrm{H} 3$ and $\mathrm{H} 4$ (ref. [23]). In principle, this complex formation is interchangeable, but seems to be differentially regulated in different cell types. For example, in HeLa cells, purified KAT7-containing HAT complex contained JADE but not BRPF [27], and BRPF-containing HAT complexes predominantly contain KAT6A/B [23]. In keeping with this, knockdown of KAT7 in HeLa cells results in reduction of all of the three acetylation marks on $\mathrm{H} 4$ (ref. [27]). In contrast, KAT7-KO in mouse embryonic fibroblasts and KAT7 knockdown in erythroblasts led to reduction specifically of $\mathrm{H} 3 \mathrm{~K} 14 \mathrm{ac}$ among the 5 potential acetylation sites and had no effect on histone H4 (ref. [24, 25]). In the present study, we found that KAT7 KO in AML cells resulted in complete loss of both $\mathrm{H} 3 \mathrm{~K} 14 \mathrm{ac}$ and H4K12ac, a pattern that has not been described before. This strongly suggests that KAT7 is solely responsible for acetylation of these two sites and that KAT6A does not compensate for KAT7 loss. It has been shown that in mouse embryos KAT6A plays a specific role in regulating local $\mathrm{H} 3 \mathrm{~K} 9$ acetylation at the Hoxa and Hoxb loci and is not involved in global H3K9 and H3K14 acetylation [41]. This might be the case in AML cells, indicating that KAT6A and KAT7 play nonoverlapping roles in histone acetylation. KAT6A also showed fitness defects in
$M L L-X$ AML cell lines in our analysis. It would be interesting to investigate the molecular basis of KAT6A dependency and the mechanisms by which cell-typespecific complex formation is regulated.

Besides the general correlation between expression level and KAT7 promoter occupancy, we found that KAT7 strongly bound to a small subset of MLL-AF9 spreading genes in MOLM-13. The expression of this subset is especially susceptible to KAT7 loss and it includes well-known leukemia maintenance genes in $M L L-X$-driven AML such as MEIS1, PBX3, JMJDIC, and MEF2C. MLL$\mathrm{X}$ target genes are known to be associated with high level of H3K79me2, which is deposited by histone methyltransferase DOT1L [8, 40]. In MLL-X spreading genes, H3K79me2 marks also spread into gene bodies, showing broader peaks [34]. These spreading genes were shown to be more sensitive to DOT1L inhibition than the other genes (non-spreading and no-bound genes); the entire set of the spreading genes are downregulated upon DOT1L inhibition [34]. This pattern of downregulation is in sharp contrast to the case of KAT7 loss, in which only a subset of the spreading genes that are highly bound by KAT7 are downregulated. Therefore, recruitment of KAT7 complex to promoters of this subset might be regulated differently from the rest of the promoters.

As a potential reader of $\mathrm{H} 3 \mathrm{~K} 14 \mathrm{ac}$ and/or H4K12ac by KAT7, we investigated BRD4 and showed that BRD4 dissociated from the promoter of the key spreading genes upon KAT7 loss. BRD4 is known to play crucial roles in maintaining $M L L-X$-driven leukemic programs $[5,6]$. A prominent role of BRD4 in AML is to activate superenhancers at the $M Y C$ locus and maintain high $M Y C$ expression [42]. Bromodomain inhibitors such as JQ1 and i-BET151 disrupt the interaction between BRD4 and acetylated histones, and evict BRD4 from chromatin, thereby leading to rapid downregulation of $M Y C$. In addition to the role at enhancers, BRD4 is known to play a role in promoting Pol II elongation at promoter-proximal regions by recruiting SEC including P-TEFb [42]. Accordingly, we observed the reduced binding of AFF1 scaffold protein of SEC upon KAT7 loss. This suggests that Pol II elongation is severely affected, causing downregulation of target genes. However, since the role of BRD4 at promoters is generic, it is still unclear why only a small subset of MLL-AF9 spreading genes are affected by KAT7 loss in MOLM-13 and why gene expression is relatively stable, even in the absence of KAT7, in the KAT7-independent OCI-AML3 cells. It is plausible that additional acetylated histone readers are involved in KAT7-dependent transcriptional activation for the MLL-AF9 spreading genes. From this perspective, it is interesting to observe that pS5-Pol II reduced its occupancy at the selected promoters. Serine 5 of Pol II is phosphorylated by TFIIH after Pol II loading onto 
promoters [43]. Subsequent Serine 2 phosphorylation on the C-terminal domain of Pol II by P-TEFb promotes Pol II elongation [43]. Therefore, KAT7-mediated histone acetylation may also be required for the initiation phase of Pol II transcription. Further studies are required to elucidate the molecular basis of KAT7-dependent transcription of MLLAF9 target genes.

Interestingly, a study by Sauer et al. [44] reported that KAT7 was downregulated in patient AML samples, yet when knocked down by shRNA, increased cell proliferation of leukemic cell lines. Analysis using a recent large-scale RNA-seq dataset [45] revealed no significant difference between CD34+ cells and AML cells (Fig. S8). Moreover, amongst 23 TCGA cancer types with matched normal samples from the same tissue as the cancer, there were only two cancer types, which showed significant differences in KAT7 expression between them, namely cholangiocarcinoma (KAT7 high in tumor) and chromophobe renal cell carcinoma (KAT7 low in tumor). These observations suggest no significant correlation between KAT7 expression levels and neoplasia or cell proliferation. In addition, during the revision of this paper, Dawson and colleagues reported that KAT7 is required for the maintenance of leukemia stem cells [46]. Their conclusion is consistent with ours and further highlights the importance of KAT7 function in leukemia maintenance.

Collectively, our findings reveal that KAT7 acts upstream of BRD4 to recruit MLL-fusion associated machineries to the promoter of key MLL-AF9 target genes, which represent an alternative therapeutic target for $M L L-X$ leukemia. We anticipate that our work will motivate the development of small-molecule inhibitors that are specific to KAT7 and highlight KAT7 as a potential therapeutic target for MLL-fusion AML.

\section{Data availability}

RNA-seq and ChIP-seq data are available from Gene Expression Omnibus under accession number GSE133516.

Acknowledgements This work was funded by the Wellcome Trust (WT206194), the Kay Kendall Leukaemia Fund (KKL920), Bloodwise (17006), Takeda Science Foundation, and Exonate Ltd. K.T. was funded by a Wellcome Trust Sir Henry Wellcome Fellowship (grant reference RG94424). G.S.V. was funded by a Cancer Research UK Senior Cancer Fellowship (C22324/A23015) and a Wellcome Trust Senior Fellowship in Clinical Science (WT095663MA). We thank Bee Ling Ng, Jennifer Graham, Christopher Hall, and Sam Thompson from the Wellcome Sanger Institute Cytometry Core Facility team for help with flow cytometry. We are grateful to the staff of the Sanger Institute Research Support Facility for help with mouse experiments and the staff of the Sanger Institute Core Sequencing facility for sequencing. We thank Mathew Garnett for providing the Nomo-1 cell line, Chris Vakoc for RN2 and MA9 cell lines, Pedro for his help compiling figures using Adobe Illustrate and Josep Nomdedeu for help advices in writing the manuscript.
Author contributions KY conceived the study and designed the experiments. YZA primarily performed the experiments and analyzed the data. M.Gu and SHO conducted bioinformatic analyses. EDB performed and analyzed the in vivo mouse studies. MG, EDB, and DA performed ChIP-qPCR and CRISPR validation studies. YT and JC performed cell proliferation assays. XC advised on ChIP experiments. JY, BJPH, MG, and KT helped with data interpretation and direction. YZA, MG, JY, GSV, and KY wrote the manuscript. All authors reviewed the manuscript.

\section{Compliance with ethical standards}

Conflict of interest GSV is a consultant for Kymab and Oxstem. All the remaining authors declare no conflict of interest.

Publisher's note Springer Nature remains neutral with regard to jurisdictional claims in published maps and institutional affiliations.

Open Access This article is licensed under a Creative Commons Attribution 4.0 International License, which permits use, sharing, adaptation, distribution and reproduction in any medium or format, as long as you give appropriate credit to the original author(s) and the source, provide a link to the Creative Commons license, and indicate if changes were made. The images or other third party material in this article are included in the article's Creative Commons license, unless indicated otherwise in a credit line to the material. If material is not included in the article's Creative Commons license and your intended use is not permitted by statutory regulation or exceeds the permitted use, you will need to obtain permission directly from the copyright holder. To view a copy of this license, visit http://creativecommons. org/licenses/by/4.0/.

\section{References}

1. Papaemmanuil E, Gerstung M, Bullinger L, Gaidzik VI, Paschka P, Roberts ND, et al. Genomic classification and prognosis in acute myeloid leukemia. N Engl J Med. 2016;374:2209-21.

2. Saultz JN, Garzon R. Acute myeloid leukemia: a concise review. J Clin Med Res. 2016;5:33.

3. Krivtsov AV, Armstrong SA. MLL translocations, histone modifications and leukaemia stem-cell development. Nat Rev Cancer. 2007;7:823-33.

4. Winters AC, Bernt KM. MLL-rearranged leukemias-an update on science and clinical approaches. Front Pediatr. 2017;5:4.

5. Dawson MA, Prinjha RK, Dittmann A, Giotopoulos G, Bantscheff $\mathrm{M}$, Chan W-I, et al. Inhibition of BET recruitment to chromatin as an effective treatment for MLL-fusion leukaemia. Nature. 2011; 478:529-33.

6. Zuber J, Shi J, Wang E, Rappaport AR, Herrmann H, Sison EA, et al. RNAi screen identifies Brd4 as a therapeutic target in acute myeloid leukaemia. Nature. 2011;478:524-8.

7. Berthon C, Raffoux E, Thomas X, Vey N, Gomez-Roca C, Yee K, et al. Bromodomain inhibitor OTX015 in patients with acute leukaemia: a dose-escalation, phase 1 study. Lancet Haematol. 2016;3:e186-95.

8. Daigle SR, Olhava EJ, Therkelsen CA, Majer CR, Sneeringer CJ, Song J, et al. Selective killing of mixed lineage leukemia cells by a potent small-molecule DOT1L inhibitor. Cancer Cell. 2011;20:53-65.

9. Daigle SR, Olhava EJ, Therkelsen CA, Basavapathruni A, Jin L, Boriack-Sjodin PA, et al. Potent inhibition of DOT1L as treatment of MLL-fusion leukemia. Blood. 2013;122:1017-25.

10. Grembecka J, He S, Shi A, Purohit T, Muntean AG, Sorenson RJ, et al. Menin-MLL inhibitors reverse oncogenic activity of MLL fusion proteins in leukemia. Nat Chem Biol. 2012;8:277-84. 
11. Tzelepis K, Koike-Yusa H, De Braekeleer E, Li Y, Metzakopian E, Dovey OM, et al. A CRISPR dropout screen identifies genetic vulnerabilities and therapeutic targets in acute myeloid leukemia. Cell Rep. 2016;17:1193-205.

12. Wang T, Yu H, Hughes NW, Liu B, Kendirli A, Klein K, et al. Gene essentiality profiling reveals gene networks and synthetic lethal interactions with oncogenic Ras. Cell. 2017;168:890-903.e15.

13. Tzelepis K, De Braekeleer E, Aspris D, Barbieri I, Vijayabaskar MS, Liu W-H, et al. SRPK1 maintains acute myeloid leukemia through effects on isoform usage of epigenetic regulators including BRD4. Nat Commun. 2018;9:5378.

14. Sheikh BN, Akhtar A. The many lives of KATs-detectors, integrators and modulators of the cellular environment. Nat Rev Genet. 2019;20:7-23.

15. Voss AK, Thomas T. MYST family histone acetyltransferases take center stage in stem cells and development. Bioessays. 2009; 31:1050-61.

16. Avvakumov N, Côté J. The MYST family of histone acetyltransferases and their intimate links to cancer. Oncogene. 2007; 26:5395-407.

17. Carapeti M, Aguiar RC, Goldman JM, Cross NC. A novel fusion between MOZ and the nuclear receptor coactivator TIF2 in acute myeloid leukemia. Blood. 1998;91:3127-33.

18. Kitabayashi I, Aikawa Y, Yokoyama A, Hosoda F, Nagai M, Kakazu N, et al. Fusion of MOZ and p300 histone acetyltransferases in acute monocytic leukemia with a $\mathrm{t}(8 ; 22)(\mathrm{p} 11 ; \mathrm{q} 13)$ chromosome translocation. Leukemia. 2001;15:89-94.

19. Panagopoulos I, Fioretos T, Isaksson M. Fusion of the MORF and CBP genes in acute myeloid leukemia with the $\mathrm{t}(10 ; 16)(\mathrm{q} 22 ; \mathrm{p} 13)$. Mol Genet. 2001;10:395-404.

20. Esteyries S, Perot C, Adelaide J, Imbert M, Lagarde A, Pautas C, et al. NCOA3, a new fusion partner for MOZ/MYST3 in M5 acute myeloid leukemia. Leukemia. 2008;22:663-5.

21. Borrow J, Stanton VP Jr, Andresen JM, Becher R, Behm FG, Chaganti RS, et al. The translocation $\mathrm{t}(8 ; 16)(\mathrm{p} 11 ; \mathrm{p} 13)$ of acute myeloid leukaemia fuses a putative acetyltransferase to the CREBbinding protein. Nat Genet. 1996;14:33-41.

22. Valerio DG, Xu H, Chen C-W, Hoshii T, Eisold ME, Delaney C, et al. Histone acetyltransferase activity of MOF is required for MLL-AF9 leukemogenesis. Cancer Res. 2017;77:1753-62.

23. Lalonde M-E, Avvakumov N, Glass KC, Joncas F-H, Saksouk N, Holliday M, et al. Exchange of associated factors directs a switch in HBO1 acetyltransferase histone tail specificity. Genes Dev. 2013;27:2009-24.

24. Mishima Y, Miyagi S, Saraya A, Negishi M, Endoh M, Endo TA, et al. The Hbo1-Brd1/Brpf2 complex is responsible for global acetylation of $\mathrm{H} 3 \mathrm{~K} 14$ and required for fetal liver erythropoiesis. Blood 2011;118:2443-53.

25. Kueh AJ, Dixon MP, Voss AK, Thomas T. HBO1 is required for H3K14 acetylation and normal transcriptional activity during embryonic development. Mol Cell Biol. 2011;31:845-60.

26. Feng Y, Vlassis A, Roques C, Lalonde M-E, González-Aguilera C, Lambert J-P, et al. BRPF3-HBO1 regulates replication origin activation and histone H3K14 acetylation. EMBO J. 2016;35:176-92.

27. Doyon Y, Cayrou C, Ullah M, Landry A-J, Côté V, Selleck W, et al. ING tumor suppressor proteins are critical regulators of chromatin acetylation required for genome expression and perpetuation. Mol Cell. 2006;21:51-64.

28. Tarumoto Y, Lu B, Somerville TDD, Huang Y-H, Milazzo JP, $\mathrm{Wu} \mathrm{XS}$, et al. LKB1, salt-inducible kinases, and MEF2C are linked dependencies in acute myeloid leukemia. Mol Cell. 2018; 69:1017-1027.e6.

29. Shi J, Wang E, Milazzo JP, Wang Z, Kinney JB, Vakoc CR. Discovery of cancer drug targets by CRISPR-Cas9 screening of protein domains. Nat Biotechnol. 2015;33:661-7.

30. Wunderlich M, Mizukawa B, Chou F-S, Sexton C, Shrestha M, Saunthararajah Y, et al. AML cells are differentially sensitive to chemotherapy treatment in a human xenograft model. Blood. 2013;121:e90-7.

31. Foy RL, Song IY, Chitalia VC, Cohen HT, Saksouk N, Cayrou C, et al. Role of Jade-1 in the histone acetyltransferase (HAT) HBO1 complex. J Biol Chem. 2008;283:28817-26.

32. Thorne RMW, Milne TA. Dangerous liaisons: cooperation between Pbx3, Meis1 and Hoxa9 in leukemia. Haematologica. 2015;100:850-3.

33. Chen M, Zhu N, Liu X, Laurent B, Tang Z, Eng R, et al. JMJD1C is required for the survival of acute myeloid leukemia by functioning as a coactivator for key transcription factors. Genes Dev. 2015;29:2123-39.

34. Kerry J, Godfrey L, Repapi E, Tapia M, Blackledge NP, Ma H, et al. MLL-AF4 spreading identifies binding sites that are distinct from super-enhancers and that govern sensitivity to DOT1L inhibition in leukemia. Cell Rep. 2017;18:482-95.

35. Nishimura K, Fukagawa T, Takisawa H, Kakimoto T, Kanemaki M. An auxin-based degron system for the rapid depletion of proteins in nonplant cells. Nat Methods. 2009;6:917-22.

36. Yokoyama A, Somervaille TCP, Smith KS, Rozenblatt-Rosen O, Meyerson M, Cleary ML. The menin tumor suppressor protein is an essential oncogenic cofactor for MLL-associated leukemogenesis. Cell. 2005;123:207-18.

37. Yokoyama A, Cleary ML. Menin critically links MLL proteins with LEDGF on cancer-associated target genes. Cancer Cell. 2008;14:36-46.

38. Muntean AG, Tan J, Sitwala K, Huang Y, Bronstein J, Connelly JA, et al. The PAF complex synergizes with MLL fusion proteins at HOX loci to promote leukemogenesis. Cancer Cell. 2010;17:609-21.

39. Ferrara F, Schiffer CA. Acute myeloid leukaemia in adults. Lancet. 2013;381:484-95.

40. Bernt KM, Zhu N, Sinha AU, Vempati S, Faber J, Krivtsov AV, et al. MLL-rearranged leukemia is dependent on aberrant H3K79 methylation by DOT1L. Cancer Cell. 2011;20:66-78.

41. Voss AK, Collin C, Dixon MP, Thomas T. Moz and retinoic acid coordinately regulate $\mathrm{H} 3 \mathrm{~K} 9$ acetylation, Hox gene expression, and segment identity. Dev Cell. 2009;17:674-86.

42. Shi J, Vakoc CR. The mechanisms behind the therapeutic activity of BET bromodomain inhibition. Mol Cell. 2014;54:728-36.

43. Brookes E, Pombo A. Modifications of RNA polymerase II are pivotal in regulating gene expression states. EMBO Rep. 2009;10: 1213-9.

44. Sauer T, Arteaga MF, Isken F, Rohde C, Hebestreit K, Mikesch J-H, et al. MYST2 acetyltransferase expression and Histone H4 Lysine acetylation are suppressed in AML. Exp Hematol. 2015;43:794-802.e4.

45. Lavallée V-P, Baccelli I, Krosl J, Wilhelm B, Barabé F, Gendron $\mathrm{P}$, et al. The transcriptomic landscape and directed chemical interrogation of MLL-rearranged acute myeloid leukemias. Nat Genet. 2015;47:1030-7.

46. MacPherson L, Anokye J, Yeung MM, Lam EYN, Chan Y-C, Weng C-F, et al. HBO1 is required for the maintenance of leukaemia stem cells. Nature. 2020;577:266-70. 